\title{
Filosofía y psicología: ¿Nuevamente juntas?
}

Ricardo Braun

Se examina la relación entre la filosofia y la psicología en la historia de la psicología contemporánea, señalando que ha existido una notable coincidencia en los temas y preocupaciones entre la filosofia de la psicología y la psicología teórica. El psicoanálisis ha sido también un tema importante en la preocupación filosófica, tanto por sus presuposiciones teóricas como por su metodología en relación con las demás ciencias. En nuestros días la unión de la psicología y la filosofia, además de otras disciplinas, se ha dado en la ciencia cognitiva.

Filosofía de la psicología / filosofía de la mente / psicología teórica

\section{Philosophy and psychology: Once again together?}

The relationship between philosophy and psychology in the history of contemporary psychology is examined, pointing out the outstanding coincidence of topics and worries found in the philosophy of mind and theoretical psychology. Psychoanalysis has also been a subject of philosophical interest, not only theoretical presuppositions of psychoanalysis but its methodology in relation to other sciences as well. Nowadays, unity between psychology and philosophy together with other disciplines, has been achieved in cognitive science.

Philosophical psychology / philosophy of mind / theoretical psychology

Correo electrónico: rbraun@correo.ulima.edu.pe 
En una carta dirigida a los miembros de la incipiente Asociación Americana de Psicología (APA, por su sigla en inglés) en 1899, Charles Bliss exhortaba a sus colegas a no formar una división en la Asociación para tener una sección dedicada al estudio de temas filosóficos. Por más que veía como importante la discusión de estos temas, la separación de filósofos en otra sección empobrecería la comprensión de los problemas centrales de la psicología. Argumentando por la unidad de la psicología y filosofía sostenía:

Nuestros mejores psicólogos están entre los mejores filósofos, y su exclusión siquiera de una parte de las reuniones de la Asociación sería una grave pérdida. Y al mismo tiempo, la mayor necesidad de la psicología en el presente es más de una sólida filosofía, y la mayor necesidad de la filosofía es más de una sólida psicología. Se debiera desear una unión más cercana que una separación posterior (Bliss, 1899).

La historia de la psicología en el siglo XX, sin embargo, no ha seguido tal deseo, al punto de que en la mayoría de casos los departamentos de psicología y filosofía de las grandes universidades se han encontrado separados por temas e intereses.

Podríamos preguntarnos si existen razones para considerar importante la filosofía en la psicología contemporánea. En la introducción a su obra Filosofia de la psicología, Bunge \& Ardila sostienen que "lo sepan o no, les guste o no, los psicólogos se basan en y utili- zan una cantidad de ideas filosóficas, sobre todo ideas acerca de la naturaleza de la mente y la ciencia. Todo psicólogo, por tanto, no solo es un científico o un terapeuta, sino un filósofo aficionado, en general malgré lui" (1987: 20). La mayoría de estudiantes de psicología no requiere cursos específicos de filosofía de la psicología o filosofía de la mente para obtener el título correspondiente. Y lo mismo se aplica a los psicólogos profesionales: el estudio de la filosofía no constituye un requisito para el ejercicio de la profesión. Sin embargo, si la tesis de Bunge \& Ardila es cierta, ¿podríamos afirmar que la filosofía de la psicología es relevante para la psicología profesional?

Considero que en vez de asumir que tácitamente los psicólogos utilizan categorías y conceptos filosóficos, debiéramos orientar el trabajo de los psicólogos profesionales para que utilicen algunas de las herramientas tradicionales de la filosofía (particularmente lógica y análisis conceptual) en la resolución de algunos problemas centrales de la psicología teórica. Recordemos que el progreso en la ciencia ha ocurrido frecuentemente por la resolución de algunos rompecabezas conceptuales que ha significado, por un lado, articular diversos conceptos, y por otro, resolver las confusiones conceptuales vía la eliminación de conceptos ambiguos, inoperantes o innecesarios. La física aristotélica unía los conceptos de velocidad instantánea con velocidad promedio y, como demostró 
Galileo, creaba paradojas y contradicciones. En la mecánica de Newton se necesitaba resolver la noción popular de peso en una de fuerza y masa. Es verdad que los científicos pueden resolver por cuenta propia los problemas conceptuales de su disciplina, sin embargo, las habilidades analíticas en las que son entrenados los filósofos pueden permitir ver con más claridad algunos problemas, particularmente aquellos que están en las fronteras de sus propias disciplinas, por la tradicional búsqueda totalizadora de la empresa filosófica.

En el caso de la psicología, hay una diferencia con las demás ciencias en lo que concierne a la filosofía. Los problemas conceptuales de la psicología están tan emparentados con la llamada "psicología popular" (en la literatura anglosajona "folk psychology") que los problemas conceptuales que han ocupado a los filósofos por siglos son muy parecidos a los temas de interés para la psicología teórica. De hecho, como algunos sostienen, la mayoría de los problemas de la psicología teórica actual podrían ser inteligibles a los filósofos que vivieron mucho antes de la aparición de la psicología científica (Block, 1979). Tómese como ejemplo el problema de la representación mental en el debate actual. Este problema no sería del todo ajeno a filósofos que abordaron el tema de las representaciones mentales como Descartes, Locke o Hume. Aun cuando los viejos problemas tienen nueva forma, el problema sigue siendo el mismo. Hoy nos preguntamos si las representaciones mentales tienen referentes reales; si pueden tener valores de verdad, si constituyen un lenguaje sintácticamente manipulable y tienen un rol causal en la conducta, y si los lenguajes naturales constituyen los sistemas de las representaciones mentales. Este acercamiento a los problemas de la psicología no ha sucedido en otras disciplinas como la física o la biología. Estoy seguro de que Aristóteles sería el primer sorprendido en conocer las investigaciones en física de partículas o en biología molecular y no tanto acaso de que sigamos preguntándonos por la naturaleza de lo mental.

En lo que sigue quiero mostrar algunos ejemplos de estudios filosóficos de la psicología que considero que han tenido influencia en la manera de abordar los problemas en la psicología del pasado y la contemporánea. Empezaré por los análisis del concepto de lo mental, del contenido mental, y finalizaré con una exposición sobre el desarrollo de la ciencia cognitiva. La ciencia cognitiva ha demostrado hasta el momento que la inclusión de la filosofía en la psicología ha sido enormemente productiva, a tal punto que ambas, y con la ayuda de otras disciplinas, han permitido entender mejor los fenómenos cognitivos y reconceptualizar el modelo de la mente. Capítulo aparte merecerá un breve comentario acerca del interés filosófico en el psicoanálisis. 


\section{DUALISMO}

El dualismo como concepción de lo mental ha sido muy influyente en la manera de entender los llamados "fenómenos mentales". Toda doctrina dualista sostiene que los seres humanos (y quizás otros seres) somos una composición de dos entidades, una primera, corpórea, física, y una segunda, no-corpórea y no-física. Esta segunda entidad sería responsable de nuestra función como seres pensantes y sensientes. La entidad corpórea tiene las propiedades de las cosas físicas, como pueden ser la espacialidad y la materialidad; la incorpórea posee propiedades mentales, a saber: el pensar y sentir.

El lenguaje popular, que ha sido considerablemente absorbido en la psicología profesional, utiliza un gran número de atribuciones tomando como referencia una concepción dualista. Por ejemplo, hablamos de "enfermedades psicosomáticas", "enfermedades mentales", "estados de ánimo", "capacidades mentales", etcétera. Bien podría ser simplemente un uso de un lenguaje enraizado culturalmente, pero lo cierto es que se emplea en las categorías de la psicología científica. Podríamos preguntarnos si los psicólogos que utilizan categorías de la llamada "psicología mental o mentalista" suscribirían a una forma de dualismo de la realidad, es decir, si creen que existen entidades mentales, que son no materiales, o el lenguaje que usan es solo una façon de parler. Pero precisamente, el lenguaje popular no tiene por qué reflejar la realidad. Después de todo, tenemos muchas concepciones populares que escasamente reflejan cómo son las cosas (la física popular puede establecer un principio como "todo lo que sube baja" que sería de manera patente falso en la física científica).

La crítica al dualismo ha ocupado mucho de la discusión de lo mental a través de la historia de la filosofía. La mayoría de las críticas al dualismo se centran en el problema de la interacción entre lo mental y lo corpóreo (o lo físico). El dualista debe probar cómo es posible que lo mental pueda ser causalmente eficiente en lo material, puesto que los efectos de lo mental suelen tener una manifestación corpórea ("escucho lo que estás diciendo, luego que lo pensaste"). Sin embargo, la posición dualista ha tenido gran dificultad para dar cuenta de esas interacciones causales sin tener que recurrir, en último término, a una explicación misteriosa que rompería todo nuestro esquema de explicación naturalista de las cosas.

La ciencia desde la era moderna ha tratado de tener una imagen coherente del mundo, y ha buscado lo que se denomina una explicación naturalista. Sin embargo, una explicación que suponga la interacción de algo no físico en el mundo físico crearía problemas en suposiciones de la física, por ejemplo, en el principio de la conservación de la masa/energía (si las mentes inmateriales tu- 
vieran efecto causal, ¿aumentaría la energía del universo al producir un efecto en el cuerpo material?). Siempre es posible considerar a la psicología como discontinua frente a las demás fuentes de conocimiento del mundo, pero inevitablemente, una psicología dualista tendría que enfrentarse a otras explicaciones, como la de la evolución biológica y dar respuesta a la aparición discontinua de mentes inmateriales.

Las críticas al dualismo tuvieron efecto particularmente en el desarrollo de la concepción conductista y funcionalista de la mente a mediados del siglo XX.

\section{CONDUCTISMO LÓGICO}

El conductismo lógico está asociado a Gilbert Ryle y Carl Hempel. En su influyente libro El concepto de lo mental, Ryle (1949) sostiene que constituye un error hablar de lo mental como si fuera una serie de causas internas de un agente en particular. El error de tipo lógico, "categorial", se crea cuando se confunde una categoría concreta por una categoría abstracta: lo que existe concretamente son las disposiciones para la conducta y a esas disposiciones las hemos llamado "mente", creyendo que la mente es algo diferente a todo el conjunto de disposiciones. En realidad, lo concreto son las disposiciones y lo abstracto la suma de disposiciones, de la misma forma como una "universidad" (categoría abstracta) es el conjun- to de edificios, biblioteca, científicos, académicos, laboratorios (categoría concreta). La "universidad" no es una cosa más de los objetos de la categoría concreta. Es un concepto totalizador de todos los objetos concretos mencionados. Entonces, para Ryle nuestro lenguaje mental debe ser entendido como una manera de hablar acerca de las disposiciones para comportarnos y los patrones de la conducta humana. La mente no es una cosa, como si fuera un "fantasma en una máquina" corporal, sino simplemente una manera de entender la suma de esas disposiciones.

Lo interesante del aporte de Ryle es que, a pesar de las claras deficiencias que se han señalado al conductismo lógico, proporciona un novedoso análisis del concepto de lo mental, considerándolo una categoría abstracta para referirse a procesos y objetos concretos. En otras palabras, le asigna a lo mental una categoría ontológica dependiente de lo conductual. Hablar de lo mental fuera de lo conductual provocaría el mismo error de atribuirle una existencia independiente al "contribuyente promedio del Estado" aparte del promedio aritmético de todos los contribuyentes del Estado.

Los trabajos de Hempel (1980), aunque más identificados con una tesis epistemológica, tienen claras pretensiones lógicas, puesto que propugnan la posibilidad de traducción, definición, análisis o reemplazo de las proposiciones mentales por proposiciones 
conductuales o ambientales sin perder su poder explicativo. Hempel, aplicando los principios de verificación del Círculo de Viena, intentaba proponer criterios lógicos del vocabulario de psicología para adecuarse a las exigencias epistemológicas determinadas por este grupo intelectual de principios del siglo XX. En efecto, toda proposición psicológica que fuera significativa debía ser especificable en proposiciones que satisficieran condiciones de observación pública. El criterio de verificación tenía que ser público, en el sentido de verificación intersubjetiva.

Las conclusiones del Círculo de Viena han tenido ataques por varios frentes y hoy en día muy pocos filósofos estarían dispuestos a defender las tesis originales del Círculo. Sin embargo, se puede entender la motivación que Hempel y otros tenían al presentar sus ideas. Se quería que la psicología fuese una ciencia, y eso suponía que contara con un conjunto de proposiciones que tuvieran un significado público y compartible que pudiera servir como un vehículo de comunicación intersubjetiva. Si las proposiciones psicológicas fueran públicas tendrían que satisfacer algunas condiciones de acceso intersubjetivo y para Hempel y otros solo las condiciones conductuales y físicas podían satisfacer el criterio de acceso público.

Esta concepción del acceso público se desarrolló considerablemente en la tesis del lenguaje privado de L. Witt- genstein (1953). Un lenguaje privado estaría compuesto por palabras individuales que solo podrían ser conocidas por la persona que habla y se referirían a sus sensaciones privadas inmediatas. Este lenguaje, por lo tanto, no podría ser entendido por otra persona. El lenguaje privado podría ser consecuencia de una doctrina dualista del tipo cartesiana, en que una persona tiene un acceso privilegiado al contenido de sus sensaciones.

Pero Wittgenstein critica la tesis del lenguaje privado porque no podría haber forma de identificar los nombres que una persona acuña con los referentes. Así, al experimentar una persona una sensación particular, le llama, supongamos, "au". El problema ocurrirá la siguiente vez que quiera aplicar la palabra "au" a una sensación igual o similar y de forma consistente. La única forma que tendría para identificar una sensación igual sería apelar a su memoria. La persona tendría que recordar la sensación original y asociarla con la sensación recurrente. Sin embargo, no tendría una instancia superior para verificar la igualdad de las sensaciones. Puesto que la persona utiliza un lenguaje privado, no puede utilizar objetos públicos para ayudarla en la identificación de su experiencia privada. Por lo tanto, no existe un criterio independiente para verificar la correcta utilización del término "au" para referirse a una sensación. Para Wittgenstein, la solución al problema de la identifica- 
ción sería consultar a un criterio público, como cuando no se está seguro de la hora de partida del tren y para verificarla apelamos a la página del horario de trenes. Pero en el caso del lenguaje privado, la sensación -que se supone que es igual-debe ser confirmada consigo misma. El ejemplo de Wittgenstein es concluyente: es como si alguien quisiera asegurarse de la veracidad de una noticia comprando varios ejemplares del mismo periódico (1953: 254).

Como sabemos, los esfuerzos de los teóricos del conductismo no pudieron contrarrestar las críticas, en especial de aquellos que cuestionaban dos suposiciones: 1) que cada predicado mental puede ser descrito de forma conductual estableciéndose una conexión lógica; y 2) que la explicación de la conducta pudiera ser agotada sin referencia a estados mentales internos o centrales.

Sobre la primera suposición fue Fodor (1968) el que desarrolló el mayor número de ataques desde el plano puramente lógico. Según Fodor las proposiciones acerca de lo mental y las proposiciones conductuales son lógicamente independientes, de tal modo que los eventos mentales pudieran ser las causas de los eventos conductuales y no que fueran lo mismo. Si fueran lógicamente dependientes la una tendría que estar conectada con la otra. Sin embargo, encontramos multiplicidad de ejemplos que demuestran que podemos tener estados internos que no resultan en una conducta observable.
Contra la segunda suposición, Davidson (1980) sostiene que un estado mental particular (como creer en algo o desear algo) solo se convierte en una acción particular cuando opera en conjunción con otros estados mentales del agente. No se podría establecer lo que hará una persona que tenga un estado mental (como una disposición en el análisis de Ryle), sin suponer otros estados mentales presentes. De modo que el análisis de un estado mental como una disposición conductual requeriría apelar a otros estados mentales. Y esto es precisamente a lo que Ryle se opone. Un estado mental no es, pues, una disposición para cierto tipo de conducta. Más bien, los estados mentales son estados internos del agente que tiene un rol causal en la producción de la conducta.

\section{LA TEORÍA DE LA IDENTIDAD}

El rechazo del dualismo y del conductismo llevó en la década de 1960 a la reconceptualización de lo mental en la forma de la llamada "teoría de la identidad". Posteriormente, la teoría de la identidad tuvo una modificación y se convirtió en la teoría funcionalista que ha influido de manera considerable en el modo de entender el problema conceptual de lo mental hasta nuestros días, como veremos más adelante.

La tesis central de la teoría de la identidad es que los estados y procesos mentales no son nada más que los esta- 
dos y procesos del cerebro. Los estados mentales son, entonces, idénticos a los estados cerebrales.

La teoría de la identidad fue desarrollada fundamentalmente por Smart (1959) y Armstrong (1965/1985), dos filósofos australianos que fueron responsables de lo que algunos denominaron "la herejía australiana" (Teichman, 1988). Esta teoría tenía una doble motivación: por un lado una postura ontológica, la concepción monista de la realidad, es decir, la suposición de que toda la realidad (incluida nuestra vida mental) está hecha de una misma sustancia, la material o física. Por otro lado, una postura metodológica, la posibilidad de la reducción del vocabulario mentalista a un vocabulario físico (al menos neurofisiológico) que esté más acorde con las ciencias físicas.

Para estos teóricos, la identidad no era de significado, es decir, la "mente" no significa "cerebro" sino solo una identidad de correlación. La teoría sostenía que había una correlación entre las sensaciones y los procesos cerebrales que eventualmente serían descritos por las neurociencias. Esta identidad estaba fundamentada en las correlaciones que empezaban a encontrarse y que se parecían a las identidades que en otras ciencias se encontraron en el pasado, a saber, que el calor es movimiento molecular, que la luz es descarga eléctrica y que el agua es $\mathrm{H}_{2} \mathrm{O}$. Como es de imaginar, esta teoría apostaba por el futuro de las neurociencias.
Sin embargo, la correlación entre mente y cerebro podía funcionar adecuadamente en algunos casos, pero parecía que en otros no. En la década de 1950 se empezaba a trabajar en la inteligencia artificial y las conclusiones de la teoría de la identidad eliminaban cualquier prospecto de atribución de algunos estados mentales a cualquier cosa que no fuera humana. Puesto que si todo evento mental es un evento neurofisiológico, entonces se excluiría cualquier arreglo físico que no fuera exactamente un evento neurofisiológico para obtener un evento mental. Así, la posibilidad de que algo no humano tuviera eventos mentales quedaba descartada. Estas reflexiones llevaron a un descontento con la teoría de la identidad originalmente formulada.

Esto, naturalmente, no supone ningún tipo de dualismo o conductismo. Lo único que se criticaba era una especie de chauvinismo cerebral para obtener eventos mentales. Podríamos fácilmente imaginarnos que existen otros seres que pudieran tener las mismas sensaciones que nosotros pero serían producidas en otros arreglos físicos. Existe evidencia de que algunas funciones asociadas a eventos mentales que el cerebro realizaba en un arreglo específico, podría hacerlo en otro arreglo, debido a una lesión en el arreglo original. De manera que sería posible que un tipo de sensación particular fuera correlacionado con diversos tipos de combinación física, incluyendo, por 
supuesto, nuestros queridos estados neurológicos.

Existe una suposición más compleja aún, que sería que un mismo pensamiento, como el estar pensando que "hace frío", podría ser el mismo pensamiento que "it's cold", o "fa fredo" y, sin embargo, por el hecho de que un hablante lo diga en diferente idioma resulte que sea una diferente instancia de estado neural.

La posibilidad de diferentes arreglos físicos para producir el mismo evento mental sugirió un cambio radical en la teoría de la identidad, de tal modo que un estado mental estaría correlacionado con una variedad diferente de tipos de estados físicos. Esta idea mantendría la tesis ontológica materialista, pero estaría comprometida con una especificación física exclusiva. Esta visión es lo que actualmente se llama la "tesis de la realizabilidad múltiple" (Putnam 1967/1975). La identidad podría mantenerse, pero una identidad contingente con varias posibilidades de la forma: comunidad filosófica y es responsable de la viabilidad de algunas de las formas del denominado "funcionalismo", que veremos a continuación.

\section{FUNCIONALISMO}

La idea intuitiva del funcionalismo es que los conceptos pueden aplicarse de acuerdo con lo que se puede hacer, como cuando decimos que un hervidor de agua "hierve agua". Es fácil ver cómo todos los artefactos a nuestro alcance están conceptualizados por su función. Un "carburador" en el auto tiene la función de distribuir el aire y la gasolina, de tal manera que el concepto "carburador" no está definido con relación a sus materiales de construcción sino a su función. Bien podría ser de acero, aluminio, plástico o el material que se crea conveniente. Lo aplicado a nuestros artefactos también se extiende a las cosas vivas. Así, tenemos que un corazón es una bomba de sangre o el ojo un órgano para ver. Como se puede inferir, la idea de la realizabilidad múltiple

Evento mental $\psi=$ evento físico $\varphi(1) \vee \varphi(2) \vee \varphi(3) \vee \varphi(4) \ldots$

De tal modo que cualesquiera de las disyunciones de lado derecho serían condición suficiente para la producción del evento mental $\psi$.

La teoría de la realizabilidad múltiple ha sido muy aceptada entre la está presente en cualquier concepción de funcionalismo.

Para la filosofía, el funcionalismo pareció proporcionar una teoría sólida que pudiera contrarrestar los problemas encontrados en el conductismo y 
en la teoría de la identidad. En efecto, el descuido del mundo interno del conductismo podría ser reemplazado por funciones causales mentales, sin que este reemplazo suponga una especificación rígida y única para proporcionar el mismo rol causal. Es como suponer que el bombeo de sangre solo se podría hacer con un corazón de tejido orgánico. De hecho, se intenta construir corazones artificiales que son funcionalmente diseñados.

Para el funcionalismo los estados mentales son estados funcionalmente descritos de acuerdo con su rol causal. Por ejemplo, el estado mental de: (1) creer que hoy es jueves puede causar que yo vaya a dictar clases porque tengo otro estado mental, (2) que creo que los jueves tengo clases, y otro (3) que creo que me descontarán si no voy a clases; y quizás otro (4), que quiero dictar clases. Ahora bien, estos estados mentales podrían causar otros estados mentales y no tan solo el dictar clases. Lo importante es que no ha habido una sola especificación física.

El funcionalismo se vio reforzado por la aparición paralela de la investigación en inteligencia artificial. Guiado por los trabajos preliminares de Turing (1950), se empezó a pensar en los fenómenos mentales como análogos con las operaciones de las máquinas computadoras. Las palabras de Turing fueron un tanto aventuradas pero eran, conceptualmente, desafiantes: "Creo que en 50 años (...) el uso de las palabras y la opinión general de la gente instruida se habrá alterado tanto que uno será capaz de máquinas pensantes sin encontrar contradicciones". El modo que defendía su pronóstico suponía el experimento del "juego de imitación". Según este juego, las habilidades de las máquinas digitales serían indistinguibles de las capacidades intelectuales humanas.

Por supuesto, después de más de 50 años no hemos evidenciado las palabras de Turing y probablemente podremos evadir el engaño que supone el juego de imitación, pero la propuesta de Turing y otros ha devenido en una serie de objeciones a la posibilidad de las máquinas pensantes de un lado, y de la posibilidad de pensamiento artificial, de otro. Esta última es explorada en nuestros días en los centros de investigación bajo el nombre de "ciencia cognitiva", sobre la que me referiré más abajo.

Las objeciones más importantes pueden ser agrupadas en dos clases: objeciones al concepto de simulación y objeciones por la diferencia entre sintaxis y semántica.

No parece ser un problema el aceptar que las computadoras "computan" pero prácticamente ningún filósofo estaría dispuesto a admitir que las computadoras estén "pensando". Lo curioso es que las computadoras simulan muy bien varios procesos asociados al mundo mental. 
En efecto, las computadoras de hoy pueden realizar complejísimos procesos de naturaleza lógica y matemática. Sin embargo, no toda simulación es la cosa misma. Veamos por qué.

Una simulación puede representar, como cuando simulamos una bolsa de valores en un laboratorio. Podemos visualizar y aprender qué pasaría en un escenario real, pero pruébese como sea, la simulación no sería la bolsa de valores misma. Nadie en su sano juicio tomaría la simulación como la verdadera bolsa de valores. Del mismo modo, una simulación de un proceso mental no necesariamente produciría un proceso mental.

Una simulación ocurre cuando un piloto se entrena en un llamado "simulador de vuelo". Las consecuencias de un error en el simulador no tienen relación con el error en un vuelo real. De manera que no es el mismo vuelo. Precisamente la diferencia entre estar en un avión y simular estarlo es la razón de ser del simulador. No se permite que el piloto aprenda directamente en el avión sino que es entrenado en algo que no es el avión. Análogamente, los objetores de la teoría funcionalista computacional afirman que cualquier parecido a una función mental por medios artificiales continúa siendo una simulación, pero no la misma cosa.

La objeción de la diferencia entre semántica y sintaxis radica en la concepción de que los procesos mentales evidencian capacidad semántica, es de- cir, capacidad para manejar significados. El clásico argumento para explicar la diferencia la proporciona Searle (1991) y su conocido argumento del "cuarto chino". Supongamos que una persona que solo conoce el idioma castellano es encerrada en un cuarto que tiene dos ventanas con las cuales se comunica con el exterior. Dentro del cuarto hay una pizarra que contiene una serie de ideogramas chinos y al costado de cada ideograma hay una palabra en castellano. La persona no sabe chino. Las personas que están afuera del cuarto utilizan el cuarto como un traductor de chino al castellano. La persona que está dentro tiene el oficio de "traductor". Por una de las ventanas los de afuera ingresan un ideograma chino esperando la traducción. El "traductor" recibe el ideograma, busca en la pizarra el símbolo y copia la palabra que está al costado. Lo escribe en un papel y lo lleva a la ventana para que sea recibido por la persona que solicitó la traducción. Searle nos invita a tener la siguiente intuición: la persona que está dentro no sabe chino, sin embargo es capaz de manipular los símbolos con facilidad. Una cosa es manipular símbolos, otra es entenderlos. Del mismo modo, una computadora trabaja con símbolos, es decir, tiene una manipulación sintáctica, pero es incapaz de entender los significados. Prueba actual de este problema la encontramos en los correctores gramaticales, que suelen ser un tanto torpes cuando se encuen- 
tran con palabras que tienen diferentes significados. Las computadoras actuales trabajan solo sintácticamente. Por ello, algunos filósofos como Searle llaman a los seres humanos "motores semánticos", a diferencia de las computadoras que serían "motores sintácticos".

Las objeciones al funcionalismo computacional ha generado un replanteamiento del funcionalismo y ha originado una metodología que está probando ser mucho más efectiva, la conjunción de fuerzas de diversas disciplinas para abordar el problema de lo mental. Este replanteamiento, que no ha sido exclusivo de las canteras filosóficas, ha llevado al desarrollo de la nueva ciencia interdisciplinaria, la ciencia cognitiva, que describiremos más adelante.

\section{PSICOANÁLISIS}

El psicoanálisis ha sido objeto de reflexión filosófica tanto desde la perspectiva de sus mismos practicantes como de sus estudiosos. Veremos a continuación algunos ejemplos que ilustran nuestro punto.

La primera relación con la filosofía tiene que ver con lo sostenido anteriormente, a saber, que el psicoanálisis es una forma de psicología popular, en el sentido de que utiliza lo que se denominan "actitudes proposicionales", ${ }^{1}$ tales como las "creencias" y los "deseos". De manera que lo que se dijo anteriormente acerca de la motivación por el análisis de los conceptos encuentra un terreno muy fértil en el psicoanálisis.

De hecho, el análisis del concepto de representación mental, que ha sido objeto del funcionalismo (Fodor, 1998) es también objeto de estudio filosófico del psicoanálisis, puesto que las actitudes proposicionales son representadas mentalmente, como cuando "se tiene una sensación acerca de algo", "se desea algo" o "se percibe algo".

Estas representaciones pueden ser lingüísticas o lingüísticamente interpretadas. Precisamente, la forma como son interpretadas las representaciones ha originado numerosas discusiones.

Otra relación con la filosofía la encontramos en las preguntas metafísicas acerca de la naturaleza de lo mental. En el seno mismo del psicoanálisis Freud mantuvo un claro interés por encontrar una solución al problema mente-cuerpo, aunque en diversas etapas de su vi-

1 El problema de las “actitudes proposicionales" fue estudiado por primera vez por G. Frege y subsecuentemente por B. Russell (1905), quien acuñó el nombre que tiene ahora. Las actitudes proposicionales más representativas son las "creencias" y "deseos" y tienen en común la "intencionalidad" que significa que toda actitud tiene un objetivo, que es la proposición que las acompaña, como, por ejemplo, "Creo que (actitud)...", donde podemos colocar una proposición como "hoy es lunes" (proposición), resultando en la actitud proposicional "Creo que hoy es lunes". 
da esta ambición tuvo cambios. Pero lo constante de su posición era la creencia en el principio de que todos los fenómenos psicológicos tenían que ser explicables en términos de una teoría biológica. Aun cuando tempranamente admitió en el Proyecto la dificultad de realizar su objetivo (Freud, 1895/19531974), nunca abandonó el principio, como cuando leemos en la obra de 1914 Sobre la historia del movimiento psico-analítico, que "todas nuestras ideas provisionales en psicología presumiblemente estarán basadas algún día en una subestructura biológica" (Freud, 1953-1974, 23: 282). Por ello, es posible afirmar que la posición filosófica de Freud con respecto al psicoanálisis era de un reduccionismo ontológico mas no metodológico. Se aceptaría la no reducción por razones de desconocimiento y estrategia, como lo afirma en Interpretación de los sueños (1900/1953-1974):

No tengo ninguna inclinación de mantener el dominio de lo psicológico flotando, como si estuviera en el aire, sin ninguna fundamentación orgánica. Pero no tengo conocimiento, ni teórico, ni terapéutico, más allá de esa convicción, de manera que me tengo que conducir como si solo tuviera lo psicológico frente a mí.

Una tercera relación importante la podemos encontrar en el campo epistemológico: la pregunta por la cientificidad del psicoanálisis. La respuesta a esta pregunta fue abordada por primera vez por el mismo Freud, quien consciente de las exigencias de los criterios de cientificidad tomados de las ciencias físicas, quería dotar al psicoanálisis de una base teórica y metodológica que pudiera cumplir con esas exigencias. Como vamos a comentar, ha habido críticas a la cientificidad del psicoanálisis desde diversos frentes, pero lo que no debiera ser objeto de disputa es la posición del mismo Freud acerca de los requisitos de lo que significaría hacer ciencia. Freud, con su larga y sólida formación en biología y neurociencia sabía de las exigencias de cualquier ciencia llamada "dura", y se podría considerar que mucho de su esfuerzo estuvo orientado a lograrlo en su teoría. En su penúltima obra, Algunas lecciones elementales de psicoanálisis, lo dice claramente: "La psicología, también, es una ciencia natural. ¿Qué más podría ser?" (1938/1953-1974, 23: 282).

En la filosofía de la ciencia, el psicoanálisis es un tema casi obligado puesto que Popper (1963) lo tomó como ejemplo, junto a las teorías de Adler y de Marx, de cómo una teoría podría ser considerada pseudocientífica por no satisfacer el denominado "criterio de demarcación". De acuerdo con Popper, las teorías científicas genuinas, tales como la teoría de la relatividad, se distinguían de las pseudocientíficas porque eran falseables, es decir, que podían someterse a test que si no los pasaban las teorías quedaban descartadas. Las teorías que no eran capaces de someterse al test eran consideradas infalseables, y por lo tanto 
pseudocientíficas. El psicoanálisis era una de esas teorías, según Popper, que era infalseable. Teorías de la ciencia posteriores a él han demostrado que su exigencia era injustificable, tomando en cuenta la historia de la ciencia y el rol de las hipótesis auxiliares. De manera que hoy en día la crítica de Popper es considerada por muchos filósofos como inadecuada y tiene poco efecto sobre nuestra concepción de lo que constituye una ciencia o no (Putnam, 1974/1991).

Una de las respuestas al desafío que planteó Popper acerca de la cientificidad del psicoanálisis fue abandonar la posición freudiana de considerar al psicoanálisis como una ciencia natural. En contraste con Freud, la tarea del psicoanálisis no sería la de explicar los fenómenos mentales en el sentido causalista que se usa en las ciencias naturales, sino de comprender los procesos mentales. Esta posición tiene sus fuentes en la taxonomía propuesta por Dilthey en psicología (1894/1977) y Jaspers en psiquiatría (1959/1962), en la que se contrasta la explicación con la comprensión. Las ciencias naturales buscan explicar utilizando leyes universales y conexiones causales generales. En cambio, la psicología (y otras disciplinas sociales o humanas) trabaja con reglas y a lo más conexiones causales particulares. De acuerdo con Jaspers, el objetivo de la psiquiatría (y psicología por extensión) es la comprensión empática de cómo un fenómeno psicológi- co puede haber emergido a partir de otro. Esta visión de las ciencias como pertenecientes o bien a la explicación o bien a la comprensión ha originado una perspectiva del psicoanálisis que generalmente se agrupa con el nombre de "herméutica" (Jaspers, 1974; Ricoeur, 1970; Habermas, 1971).

Uno de los críticos de la visión hermenéutica más prominentes en nuestros días es Grünbaum, quien proviniendo de la física y de la filosofía de la ciencia ha hecho contribuciones notables al cuestionamiento, replanteamiento y respuesta de algunos problemas del psicoanálisis. Su trabajo no solo es respetado por los críticos del psicoanálisis sino también por renombrados teóricos dentro del mismo psicoanálisis (Edelson, 1988). En contra de la posición hermenéutica Grünbaum ha sostenido que los estándares adecuados para la evaluación del psicoanálisis deben ser los derivados de la ciencia empírica, y la división propuesta por Dilthey y sus seguidores obedece a una incomprensión de los fundamentos de las ciencias físicas (Grünbaum, 1984). Este autor ha cuestionado la tesis popperiana de la infalsabilidad del psicoanálisis (Grünbaum, 1977). Considera que el psicoanálisis es una ciencia (en contra de Popper), y que es una ciencia empírica (en contra de los "hermenéuticos"). Pero, con respecto a la pregunta de si el psicoanálisis es una buena ciencia, la respuesta de Grünbaum es negativa. Mucha de su 
obra ha sido la defensa de esta última conclusión. El psicoanálisis no solo tiene problemas derivados del uso de los datos clínicos, sino también por los modos de razonamiento que Freud utilizó para proporcionar evidencia a favor de su teoría (1984).

\section{Ciencia cognitiva}

Aún es prematuro para poder identificar claramente las características de la ciencia cognitiva. Según algunos científicos, que están trabajando en departamentos con ese nombre, la ciencia cognitiva solo es la denominación que permite buscar fondos o administrar el pago de los salarios (Searle, 1995). Pero parece que hay algo en común en las convicciones de las personas involucradas: una reacción en contra de lo que fue el paradigma dominante en la psicología: el conductismo.

Ya hemos visto algunos de los problemas filosóficos del conductismo, pero las alternativas han traído otro grupo de problemas. Por ello, muchos científicos consideraron que el mejor camino para entender uno de los procesos más complejos de la mentalidad humana, la cognición, se debía de contar con la asistencia de personas de diversas especialidades. Los primeros acercamientos interdisciplinarios ocurrieron entre la naciente psicología cognitiva y la inteligencia artificial.

La ciencia cognitiva o también llamada "la nueva ciencia de la mente"
(Gardner, 1985) es, por definición, interdisciplinaria. Sus practicantes suelen asociar las disciplinas que mejor han colaborado para entender los problemas acerca del funcionamiento mental, particularmente el cognoscitivo (adquisición, almacenamiento y uso de la actividad inteligente): psicología cognitiva, filosofía, inteligencia artificial o ciencia de la computación, lingüística y neurociencia. Algunos añaden la antropología.

Los psicólogos cognitivos están interesados en entender las capacidades mentales, como la atención y la memoria; los lingüistas se dedican al estudio de la estructura del lenguaje humano y la naturaleza de la adquisición del lenguaje; los filósofos están dedicados a la lógica y la búsqueda de sentido, y a la aclaración conceptual de términos como la información y el conocimiento; los científicos de la computación quieren desarrollar la inteligencia artificial y, finalmente, los neurocientíficos estudian la organización del sistema nervioso y su función.

\section{Papel de la filosofía en la CienCia COGNITIVA}

Como se sabe, la filosofía es una disciplina que estudia los fundamentos de las cosas. En ese sentido, no solo puede ayudar a establecer las bases en las que se construye el conocimiento sino, además, puede hacer un seguimiento de la justificación del conocimiento confor- 
me se crea. Los filósofos pueden ayudar a los científicos a clarificar el objeto de estudio, la normatividad de los métodos y las relaciones entre las diferentes teorías de la ciencia y teorías científicas.

De acuerdo con algunos filósofos que participan en las ciencias cognitivas, son tres las áreas fundamentales en las que aportan su particular habilidad: a) definición del trabajo, su objeto de estudio y su metodología (filosofía de la ciencia); b) análisis de las estructuras y entidades postuladas por la ciencia cognitiva y su relación con los conceptos ordinarios y el mundo físico (ontología); y c) reflexión acerca de las interrelaciones entre las representaciones y cómo las personas las organizan y usan para generar conocimiento (epistemología) (Baker-Ward, 1987).

El avance de la ciencia cognitiva es hoy en día fascinante, especialmente por la diversidad de enfoques que demuestran la complejidad de su objeto de estudio y todo hace pensar que en las siguientes generaciones, con profesionales que han sido expuestos a esta visión interdisciplinaria, puede incrementarse nuestra comprensión de los fenómenos mentales a partir de la investigación colaborativa.

Es oportuno señalar que el ideal que mencionamos simbólicamente con las palabras de Bliss se ha visto cumplido pero en mayor dimensión, puesto que no solamente están unidas la psicología y la filosofía, sino también otras disci- plinas que permiten un mejor esclarecimiento y, sin duda alguna, una posibilidad de solución al problema del fenómeno mental.

\section{REFERENCIAS}

Armstrong, D. (1965/1985). The nature of mind. En: Block, N. (Ed.). Readings in Philosophy of Psychology. Cambridge, MA: Harvard University Press.

Baker-Ward, L. (1987). Philosophy: Foundations of cognitive science. En: Stillings, N. et al. (Eds.). Cognitive science: An introduction. Cambridge, MA: The MIT Press.

Bliss, C. (1899). Proposed changes in the American Psychological Association. Psychological Review, 6, 237-238.

Block, N. (1979). Philosophy of psychology. En: Asquith, P. \& Kyburg, H. (Eds.). Current research in philosophy of science (pp. 450-462). East Lansing: Philosophy of Science Association.

Bunge, M. \& Ardila, R. (1987). Filosofia de la psicología. Barcelona: Ariel.

Davidson, D. (1980). Essays on Actions and Events. Nueva York: Oxford University Press.

Dilthey, W. (1894/1977). Descriptive psychology and historical understanding. The Hague: Martinus Nijhoff.

Edelson, M. (1988). Psychoanalysis: A theory in crisis. Chicago: University of Chicago Press.

Fodor, J. (1968). Psychological explanation. Nueva York: Random House.

Fodor, J. (1998). Cognitive Science: Where Cognitive Science Went Wrong. Oxford: Clarendon Press. 
Freud, S. (1953/1974). The standard edition of the complete psychological works of Sigmund Freud. Strachey, J. (Ed.). 23 tomos. Londres: The Hogarth Press and the Institute of PsychoAnalysis.

Gardner, H. (1985). The mind's new science: A history of the cognitive revolution. Nueva York: Basic Books.

Grünbaum, A. (1977). How scientific is psychoanalysis? En: Stern, R., Horowitz, L. \& Lunes, J. (Eds.). Science and psychotherapy. Nueva York: Haven Press.

Grünbaum, A. (1984). The foundations of psychoanalysis: A critique. Berkeley: University of California Press.

Habermas, J. (1971). Knowledge and human interests. Heinemann.

Hempel, C. (1980). The logical analysis of psychology. En: Block, N. (Ed.). Readings in philosophy of psychology (pp. 14-23). Cambridge, MA: Harvard University Press.

Jaspers, K. (1959/1963). General psychopathology. Manchester: Manchester University Press.

Jaspers, K. (1974). Causal and 'meaningful' connections between life history and psychosis. En: Hirsch, S. \& Shepard, M. (Eds.). Themes and variations in european psychiatry (pp. 80-93). Charlottesville: University of Virginia Press.

Popper, K. (1963). Conjectures and refutations: The growth of scientific knowledge. Nueva York: Harper and Row.

Putnam, H. (1967/1975). Psychological predicates. En: Putnam, H. (Ed.). Mind, language and reality: Philosophical papers. Vol. 2. Cambridge, MA: Cambridge University Press.

Putnam, H. (1974/1991). The "corroboration" of theories. En: The philosophy of science. Cambridge, MA: The MIT Press.

Reppen, J. (1985). Beyond Freud. Hillsdale, NJ: The Analytic Press.

Ricoeur, P. (1970). Freud and philosophy. New Haven: Yale University Press.

Russell, B. (1905/1975). On denoting. En: Davidson, D. \& G. Harman (Eds.). The logic of grammar. Encino: Dickenson Publishing.

Ryle, G. (1949). The concept of mind. Londres: Hutchinson.

Searle, J. (1991). Minds, brains and programs. En: Rosenthal, D. (Ed.). The nature of mind. Nueva York: Oxford University Press.

Searle, J. (1995). Ontology is the question. En: Baumgartner, P. \& Payr, S. (Eds.). Speaking minds: Interviews with twenty eminent cognitive scientists. Princeton: Princeton University Press.

Smart, J. J. C. (1959/1962). Sensations and brain processes. En: Chappell, V. C. (Ed.). The philosophy of mind. Englewood Cliffs, NJ: Prentice-Hall.

Teichman, J. (1988). Philosophy and the mind. Nueva York: Basil Blackwell.

Turing, A. (1950). Computing machinery and intelligence. Mind, 59, 433-460.

Wittgenstein, L. (1953). Philosophical investigations. Nueva York: Macmillan. 